\title{
HDAC INHIBITORS SODIUM BUTYRATE AND SODIUM VALPROATE DO NOT AFFECT HUMAN NCOR1 AND NCOR2 GENE EXPRESSION IN HL-60 CELLS
}

\author{
Jiri Vrba $^{\text {a**, Katerina Trtkova }}{ }^{\mathrm{b}}$, Jitka Ulrichova ${ }^{\mathrm{a}, \mathrm{c}}$ \\ a Department of Medical Chemistry and Biochemistry, Faculty of Medicine and Dentistry, Palacky University Olomouc, \\ Czech Republic \\ ${ }^{b}$ Laboratory of Molecular Pathology, Department of Pathology, Faculty of Medicine and Dentistry, Palacky University \\ Olomouc \\ c Institute of Molecular and Translational Medicine, Faculty of Medicine and Dentistry, Palacky University Olomouc \\ E-mail:vrbambv@seznam.cz
}

Received: March 21, 2011; Accepted: May 3, 2011

Key words: Nuclear corepressor/Histone deacetylase/NADPH oxidase/Valproic acid/All-trans retinoic acid/Gene expression/ Differentiation

Aim. This study was designed to examine whether the class I and class IIa histone deacetylase (HDAC) inhibitors, sodium butyrate and sodium valproate alter the expression of human NCOR1 and/or NCOR2 genes coding for N-CoR (nuclear receptor corepressor) and SMRT (silencing mediator for retinoid and thyroid hormone receptors), respectively.

Methods. Human leukemia HL-60 cells were treated for $24 \mathrm{~h}$ with 0.5 and $1 \mathrm{mM}$ sodium butyrate, 1 to $3 \mathrm{mM}$ sodium valproate, $1 \mathrm{mcM}$ all-trans retinoic acid (ATRA) or cotreated with $1 \mathrm{mcM}$ ATRA and $0.5 \mathrm{mM}$ sodium butyrate. The acetylation of histones $\mathrm{H} 3$ and $\mathrm{H} 4$ was analysed by western blotting. The levels of NCOR1 and NCOR2 mRNA were determined by quantitative real-time PCR. Expression of NCF2 gene coding for the NADPH oxidase subunit p67phox was evaluated as a marker of myeloid differentiation.

Results. Both butyrate and valproate increased the acetylation of histone H3 at Lys9 and/or Lys 14 as well as histone $\mathrm{H} 4$ at Lys 12. Both HDAC inhibitors caused a significant increase in NCF2 mRNA levels without affecting NCOR1 or NCOR2 mRNA levels. Similarly, ATRA alone or in combination with butyrate induced NCF2 gene expression without any significant influence on the expression of NCOR1 or NCOR2 genes.

Conclusion. We conclude that inhibitors of class I and class IIa HDACs do not alter the expression of human NCOR1 or NCOR2 genes and that the onset of myeloid differentiation is not accompanied by induction or repression of these genes in HL-60 cells.

\section{INTRODUCTION}

N-CoR (nuclear receptor corepressor) and SMRT (silencing mediator for retinoid and thyroid hormone receptors) are ubiquitously expressed corepressor proteins. They interact with unliganded nuclear receptors and form large corepressor complexes that mediate transcriptional repression through their association with histone deacetylases (HDACs). The relative hypoacetylation restores a positive charge in histone proteins and causes closer association of the histones with DNA that becomes less accessible to transcription factors ${ }^{1,2}$. HDACs that have been shown to interact with N-CoR or SMRT complexes include class I HDACs 1, 2 and 3 and class IIa HDACs 4, 5 and 7 (ref. ${ }^{3}$ ). N-CoR and SMRT have been recognized to play important roles, among others, in development and cell differentiation ${ }^{1}$. For instance, neutrophilic differentiation is regulated through the nuclear retinoic acid receptor $\alpha(\mathrm{RAR} \alpha)$. Unliganded RAR $\alpha$ interacts with $\mathrm{N}-\mathrm{CoR}$ or SMRT which associate through the corepressor mSin 3 (mammalian switch independent 3 protein) with HDAC 1 or HDAC2. The corepressor complexes, however, dissociate from RAR $\alpha$ at physiological concentrations of all-trans retinoic acid (ATRA) and subsequent recruitment of coactivators with histone acetylase activity relieves the transcriptional repression of differentiationrelated genes ${ }^{4}$. On the other hand, abnormal transcriptional repression resulting from aberrant recruitment of the N-CoR/SMRT-HDAC complexes appears to underlie the molecular pathogenesis of cancers such as acute promyelocytic leukemia ${ }^{5}$. In accordance with the crucial role of HDACs in corepressor-dependent transcriptional repression, inhibitors of HDAC activity have emerged as a new class of anticancer agents ${ }^{6}$.

Human N-CoR and SMRT are encoded by NCORI (nuclear receptor corepressor 1) and NCOR2 (nuclear receptor corepressor 2) genes, respectively. To date, little is known about their transcriptional regulation. It has been shown that the steroid hormone, 17 $\beta$-estradiol decreases $\mathrm{N}$-CoR protein levels in estrogen receptor-positive breast cancer cells without affecting NCOR1 mRNA levels ${ }^{7}$. NCOR 1 mRNA levels were also unchanged in rat pituitary $\mathrm{GH}_{3}$ cells exposed to $17 \beta$-estradiol or to the thyroid hormone triiodothyronine ${ }^{8}$. The steroid hormone, $1 \alpha, 25$-dihydroxyvitamin $\mathrm{D}_{3}$ has been found to induce a moderate and transient upregulation of NCOR2 but not NCOR1 mRNA levels in MCF-7 breast cancer cells9. Both NCOR1 and NCOR2 mRNA levels remained unaf- 
fected in rat hepatocytes treated with ATRA or colchicine, a microtubule interfering agent ${ }^{10}$.

Since HDAC inhibitors are considered to modulate the expression of $7-10 \%$ of genes in human cancer cells ${ }^{11}$, we examined whether the expression of human NCORI and/or NCOR2 genes could be altered by two HDAC inhibitors, sodium butyrate and sodium valproate.

\section{MATERIAL AND METHODS}

\section{Chemicals}

Sodium butyrate (B5887), sodium valproate (P4543) and ATRA (R2625) were obtained from Sigma-Aldrich (St. Louis, MO, USA). Stock solutions of sodium butyrate and sodium valproate (1 $\mathrm{M}$ ) were prepared in water for molecular biology and stored at $-20^{\circ} \mathrm{C}$. Stock solution of ATRA ( $1 \mathrm{mM})$ in ethanol was prepared freshly before use with all manipulations performed in subdued light.

\section{Cell culture and treatment}

The human promyelocytic leukemia cell line HL-60 (No. 98070106, ECACC, Salisbury, UK) was cultured at $37{ }^{\circ} \mathrm{C}$ in RPMI-1640 medium supplemented with $2 \mathrm{mM}$ L-glutamine, $100 \mathrm{U} / \mathrm{ml}$ penicillin, $100 \mu \mathrm{g} / \mathrm{ml}$ streptomycin (Sigma), and 10\% fetal bovine serum (Invitrogen, Carlsbad, CA, USA) in a humidified atmosphere containing $5 \% \mathrm{CO}_{2}$ and maintained between $1-8 \times 10^{5}$ cells $/ \mathrm{ml}$. All experiments were conducted using exponentially growing cells, passages 10-30. Cells were seeded at a density of $1.5 \times 10^{5}$ cells $/ \mathrm{ml}$ and treated for $24 \mathrm{~h}$ with butyrate, valproate or ATRA. Negative controls were treated with $0.1 \%$ ethanol or untreated.

\section{Western blot analysis}

After treatment, cells were collected by gentle centrifugation, washed with PBS and lysed in lysis buffer $(50 \mathrm{mM}$ Tris, $150 \mathrm{mM} \mathrm{NaCl}, 1 \mathrm{mM}$ EDTA, $1 \mathrm{mM} \mathrm{NaF}, 1 \mathrm{mM}$ $\mathrm{Na}_{3} \mathrm{VO}_{4}, 1 \mathrm{mM}$ phenylmethylsulfonyl fluoride, protease inhibitors Complete (Roche Diagnostics, Mannheim, Germany), $0.2 \%$ sodium dodecyl sulfate (SDS), $1 \%$ Nonidet-P40, 1\% Triton X-100, pH 7.4). After incubation on ice for $20 \mathrm{~min}$, the cell lysates were sonicated to shear genomic DNA using an Ultrasonic Processor UP200s equipped with a sonicator probe Sonotrode Microtip S2 (Hielscher, Teltow, Germany). The samples were kept on ice and sonicated for $2 \times 20 \mathrm{~s}$ at an $80 \%$ amplitude, $2 \times$ $20 \mathrm{~s}$ at a $90 \%$ amplitude and $2 \times 20 \mathrm{~s}$ at a $100 \%$ amplitude with a cycle set at $0.5 \mathrm{~s}$. After this, the cell lysates were centrifuged for $10 \mathrm{~min}$ at $16000 \mathrm{~g}$ and $4{ }^{\circ} \mathrm{C}$ and the proteins in supernatants were quantified using the Pierce BCA Protein Assay Kit (Thermo Scientific, Rockford, IL, USA). Aliquots containing $30 \mu \mathrm{g}$ of protein were subjected to electrophoresis through $12.5 \%$ SDS-polyacrylamide gel and proteins were transferred to polyvinylidene difluoride membrane by electroblotting. The membranes were probed with rabbit monoclonal histone $\mathrm{H} 3$ antibody (No. 9717), rabbit polyclonal acetyl-histone H3 (Lys9/Lys 14) antibody (No. 9677) or rabbit polyclonal acetyl-histone H4 (Lys 12) antibody (No. 2591), all purchased from Cell
Signaling Technology (Danvers, MA, USA). The primary antibodies were visualized using the Phototope-HRP Western Blot Detection System containing anti-rabbit IgG-HRP conjugated secondary antibody (Cell Signaling Technology).

\section{Reverse transcription and quantitative real-time PCR}

Total RNA was extracted using the RNeasy Mini Kit (Qiagen, Hilden, Germany) according to the manufacturer's instructions. The concentration of RNA was determined by spectrophotometry at $260 \mathrm{~nm}$. RNA samples $(2 \mu \mathrm{g})$ were reverse transcribed using the High Capacity cDNA Reverse Transcription Kit (Applied Biosystems, Foster City, CA, USA) according to the manufacturer's recommendations. Real-time PCR was performed on a LightCycler 480 II system (Roche Diagnostics) using the TaqMan Universal PCR Master Mix and the TaqMan Gene Expression Assays consisting of specific primers and FAM dye-labelled TaqMan minor groove binder probes (Applied Biosystems). The assay ID for NCOR1 was Hs00196920_m1; for NCOR2, Hs00196955_m1; for NCF2, Hs00166416_m1; and for 18S, Hs99999901_s1, respectively. Amplification conditions were $50{ }^{\circ} \mathrm{C}$ for $2 \mathrm{~min}$, $95^{\circ} \mathrm{C}$ for $10 \mathrm{~min}$, followed by 40 cycles with $95^{\circ} \mathrm{C}$ for $15 \mathrm{~s}$ and $60{ }^{\circ} \mathrm{C}$ for $1 \mathrm{~min}$. Crossing point values, equivalent to $C_{\mathrm{T}}$, were determined automatically using the second derivative maximum analysis. Relative changes in gene expression were calculated by the comparative $C_{\mathrm{T}}$ method using the $2^{-\Delta \Delta C}$ equation with results normalized to $18 \mathrm{~S}$ rRNA levels.

\section{Statistical analysis}

Results were expressed as means \pm S.D. of three independent experiments. The differences in mean values were analysed by Student's $t$-test. A $p$ value of less than 0.05 was considered as statistically significant.

\section{RESULTS}

\section{Hyperacetylation of histones $\mathrm{H} 3$ and $\mathrm{H} 4$ by butyrate and valproate}

We chose for our study non-cytotoxic concentrations of butyrate and valproate on the basis of our previous results $^{12}$. To confirm efficient inhibition of HDAC activity, human leukemia HL-60 cells were treated with 0.5 and $1 \mathrm{mM}$ butyrate or 1 to $3 \mathrm{mM}$ valproate and the whole cell lysates were analysed by western blotting using antibodies detecting specific acetylations of histones $\mathrm{H} 3$ and $\mathrm{H} 4$. After $24 \mathrm{~h}$ treatment, both butyrate and valproate increased the acetylation of histone $\mathrm{H} 3$ at Lys9 and/or Lys 14 as well as the acetylation of histone H4 at Lys 12 (Fig. 1).

\section{Effect of butyrate and valproate on NCOR1, NCOR2 and NCF2 gene expression}

The exposure of HL-60 cells to butyrate or valproate is associated with multiple changes in gene expression leading to monocytic differentiation ${ }^{13,14}$. We examined the expression of human NCOR1 and NCOR2 genes encoding 


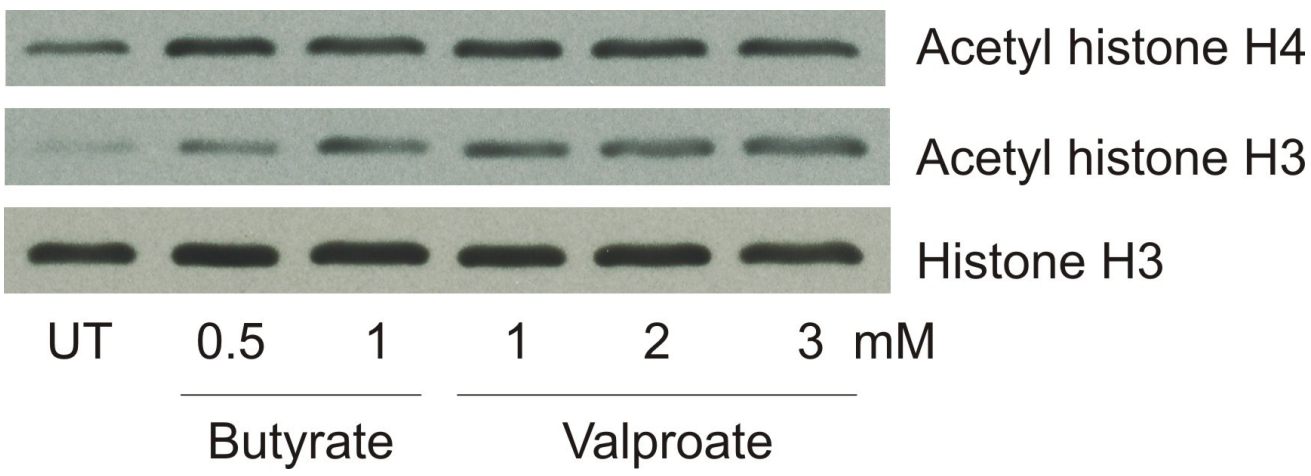

Fig. 1. Hyperacetylation of histones by sodium butyrate and sodium valproate in HL-60 cells. HL-60 cells were treated for $24 \mathrm{~h}$ with butyrate, valproate or untreated (UT). Proteins in the whole cell lysates ( $30 \mu \mathrm{g} / \mathrm{lane}$ ) were analysed by western blotting using antibodies against acetylated histone $\mathrm{H} 4$ (Lys12), acetylated histone H3 (Lys9/ Lys 14) and total histone H3. The figures are representative of two experiments.

N-CoR and SMRT, respectively, concurrently with the expression of NCF2 (neutrophil cytosolic factor 2) gene coding for the NADPH oxidase subunit p67phox, a marker of myeloid differentiation ${ }^{12,15}$. After $24 \mathrm{~h}$ treatment of HL-60 cells, both butyrate and valproate induced a dosedependent increase in NCF2 gene expression as shown by the real-time PCR technique (Fig. 2A). In contrast, neither butyrate nor valproate caused any substantial changes in the expression of NCOR1 or NCOR2 genes under the same conditions. The NCOR 1 mRNA levels induced by butyrate or valproate reached $(0.83 \pm 0.09)$-fold to $(1.08 \pm$ 0.04 )-fold values, while the NCOR 2 mRNA levels varied between $(0.80 \pm 0.11)$-fold and $(1.25 \pm 0.22)$-fold values compared to control cells (Fig. 2A). We also examined the effect of the HDAC inhibitors in the presence of ATRA, an inducer of neutrophilic differentiation ${ }^{13}$. Our preliminary experiments (data not shown) demonstrated that only $0.5 \mathrm{mM}$ butyrate synergized with ATRA in the induction of the NCF2 gene expression without parallel induction of the cytotoxic effect ${ }^{12}$. While the NCF2 mRNA levels in HL-60 cells exposed for $24 \mathrm{~h}$ to $0.5 \mathrm{mM}$ butyrate or $1 \mu \mathrm{M}$ ATRA reached 2.9-fold (Fig. 2A) and 7.2-fold values (Fig. 2B), respectively, the coexposure to ATRA and butyrate caused 17.3-fold increase in NCF2 mRNA level compared to control cells (Fig. 2B). On the other hand, ATRA either alone or in combination with butyrate did not substantially affect the expression of either NCOR1 or NCOR2 genes (Fig. 2B).

\section{DISCUSSION}

$\mathrm{N}-\mathrm{CoR}$ and SMRT are the most extensively studied corepressor proteins, but little is known about their transcriptional regulation. This study was designed to examine whether the expression of human NCOR1 and NCOR2 genes coding for N-CoR and SMRT, respectively, could be altered by two HDAC inhibitors, sodium butyrate and sodium valproate. Human HDAC enzymes are classified on the basis of their homology to yeast HDACs. Classical HDACs are $\mathrm{Zn}^{2+}$-dependent enzymes and in- clude class I (HDAC1, 2, 3, 8), class IIa (HDAC4, 5, 7, 9), class IIb (HDAC6, 10) and class IV (HDAC11). Class III enzymes require $\mathrm{NAD}^{+}$for their activity and comprise seven members known as sirtuins ${ }^{6}$. Inhibitors of HDAC activity are able to cause both induction and repression of gene expression ${ }^{16}$. These effects may result from the increased acetylation of histones and/or nonhistone HDAC substrates that include a number of transcription factors and other important proteins ${ }^{11}$. Butyrate and valproate inhibit at millimolar concentrations class I and class IIa HDACs s $^{11,16,17}$, i.e. enzymes known to associate with N-CoR and SMRT (ref. ${ }^{3}$ ). The HL-60 cell line used in our study was derived from a patient with acute myeloblastic leukemia type M2 according to the FrenchAmerican-British classification ${ }^{18}$. HL-60 cells may be induced to differentiate along the neutrophilic pathway, e.g. by ATRA, or along the monocytic pathway by butyrate ${ }^{13}$, valproate ${ }^{14}$ and other compounds. ATRA activates the transcription of differentiation-related genes through its binding to RAR $\alpha$ leading to the release of the N-CoR/ SMRT-HDAC complexes ${ }^{4}$. Butyrate and valproate may induce expression of the same genes as ATRA by inhibiting HDACs in the corepressor complexes ${ }^{5}$. Moreover, a combination of HDAC inhibitors with ATRA has been shown to synergize in differentiation of ATRA-sensitive myeloid leukemic cells ${ }^{19}$ and to overcome the block in differentiation of ATRA-resistant cells ${ }^{20}$. In this study, we found that neither butyrate nor valproate, like ATRA, induced any significant changes in the expression of NCORI and NCOR2 genes under conditions of efficient HDAC inhibition and/or induction of myeloid gene expression. We conclude that the inhibitors of class I and class IIa HDACs do not alter the expression of human NCORI and $N C O R 2$ genes and that the onset of myeloid differentiation is not accompanied by induction or repression of these genes in HL-60 cells. 
A

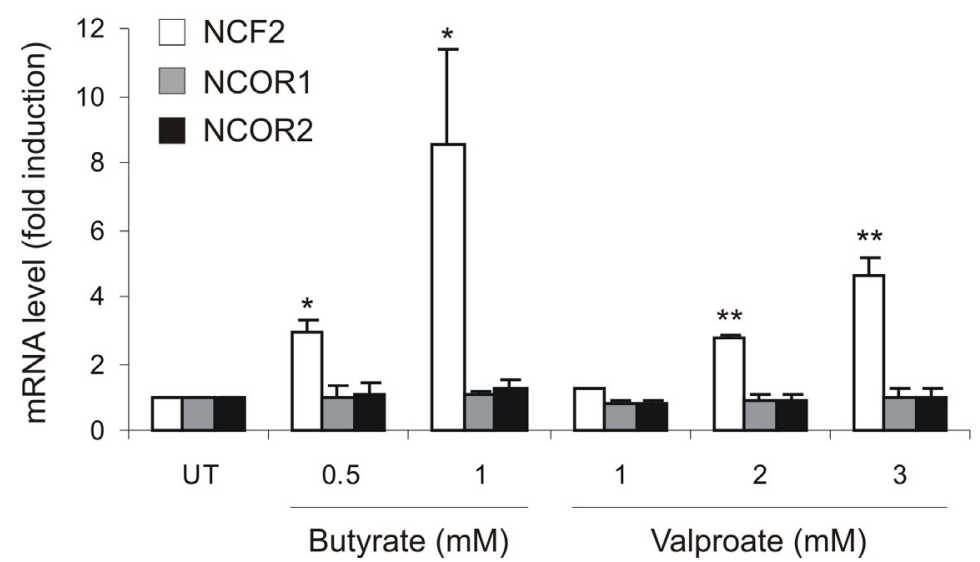

B

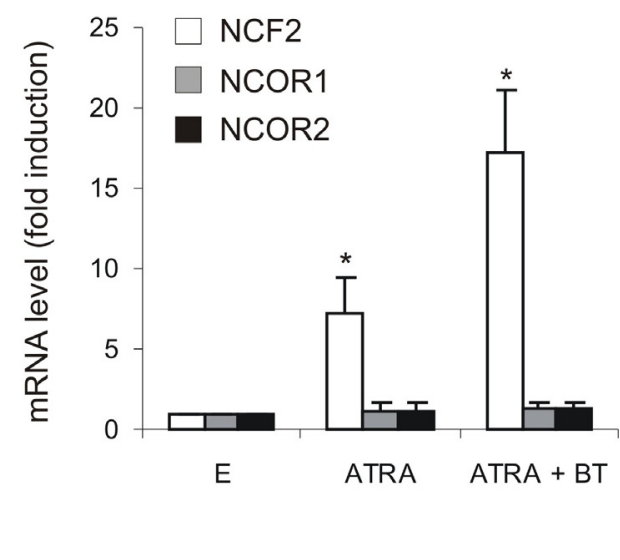

Fig. 2. Relative changes in NCOR1, NCOR2 and NCF2 gene expression by sodium butyrate, sodium valproate and all-trans retinoic acid (ATRA) in HL-60 cells. (A) HL-60 cells were treated for $24 \mathrm{~h}$ with butyrate, valproate or untreated (UT; control). (B) HL-60 cells were treated for $24 \mathrm{~h}$ with $0.1 \%$ ethanol (E; control), $1 \mu \mathrm{M}$ ATRA or cotreated with $1 \mu \mathrm{M}$ ATRA and $0.5 \mathrm{mM}$ butyrate (BT). The levels of NCOR1, NCOR2 and NCF2 mRNA levels were determined by quantitative real-time PCR with results normalized to $18 \mathrm{~S}$ rRNA levels. Data are means $\pm \mathrm{SD}$ of three experiments. * $p<0.05 ; * * p<0.01$, significantly different from control.

\section{ABBREVIATIONS}

ATRA, All-trans retinoic acid; HDAC, Histone deacetylase; NCF2, Neutrophil cytosolic factor 2; N-CoR, Nuclear receptor corepressor; NCOR1, Nuclear receptor corepressor 1; NCOR2, Nuclear receptor corepressor 2; RAR $\alpha$, Retinoic acid receptor $\alpha$; SMRT, Silencing mediator for retinoid and thyroid hormone receptors

\section{ACKNOWLEDGEMENTS}

This work was supported by grants from the Ministry of Education, Youth and Sports of the Czech Republic (Nos. MSM 6198959216 and CZ.1.05/2.1.00/01.0030)

\section{REFERENCES}

1. Auger AP, Jessen HM. Corepressors, nuclear receptors, and epigenetic factors on DNA: a tail of repression. Psychoneuroendocrinology 2009;34 Suppl 1:S39-47.

2. Karagianni P, Wong J. HDAC3: taking the SMRT-N-CoRrect road to repression. Oncogene 2007;26:5439-5449.

3. Stanya KJ, Kao HY. New insights into the functions and regulation of the transcriptional corepressors SMRT and N-CoR. Cell Div 2009; $4: 7$.

4. Ozpolat B. Acute promyelocytic leukemia and differentiation therapy: molecular mechanisms of differentiation, retinoic acid resistance and novel treatments. Turk J Hematol 2009;26:47-61.

5. Minucci S, Nervi C, Lo Coco F, Pelicci PG. Histone deacetylases: a common molecular target for differentiation treatment of acute myeloid leukemias? Oncogene 2001;20:3110-3115.

6. Witt O, Deubzer HE, Milde T, Oehme I. HDAC family: What are the cancer relevant targets? Cancer Lett 2009;277:8-21.

7. Frasor J, Danes JM, Funk CC, Katzenellenbogen BS. Estrogen down-regulation of the corepressor N-CoR: mechanism and implications for estrogen derepression of N-CoR-regulated genes. Proc Natl Acad Sci U S A 2005;102:13153-13157.
8. Misiti S, Schomburg L, Yen PM, Chin WW. Expression and hormonal regulation of coactivator and corepressor genes. Endocrinology 1998;139:2493-2500.

9. Dunlop TW, Vaisanen S, Frank C, Carlberg C. The genes of the coactivator TIF2 and the corepressor SMRT are primary $1 \alpha, 25(\mathrm{OH})_{2} \mathrm{D}_{3}$ targets. J Steroid Biochem Mol Biol 2004;89-90:257260.

10. Macejova D, Dvorak Z, Vrzal R, Ulrichova J, Ondkova S, Brtko $\mathrm{J}$. The effect of all-trans retinoic acid and/or colchicine on expression of rexinoid and thyroid hormone nuclear receptors and their coregulators in primary rat hepatocytes. Gen Physiol Biophys 2007;26:240-242.

11. $\mathrm{Xu}$ WS, Parmigiani RB, Marks PA. Histone deacetylase inhibitors: molecular mechanisms of action. Oncogene 2007;26:5541-5552.

12. Vrba J, Dolezel P, Ulrichova J. Neutrophilic differentiation modulates the apoptotic response of HL-60 cells to sodium butyrate and sodium valproate. Neoplasma 2010;57:438-448.

13. Collins SJ. The HL-60 promyelocytic leukemia cell line: proliferation, differentiation, and cellular oncogene expression. Blood 1987;70:1233-1244.

14. Cheng YC, Lin H, Huang MJ, Chow JM, Lin S, Liu HE. Downregulation of c-Myc is critical for valproic acid-induced growth arrest and myeloid differentiation of acute myeloid leukemia. Leuk Res 2007;31:1403-1411.

15. Miyamoto D, Someya A, Nunoi H, Nagaoka I, Yamashita T. Analysis of the NADPH oxidase components during differentiation of HL-60 cells to eosinophilic lineage. Biochim Biophys Acta 1994; 1224:11-16.

16. Davie JR. Inhibition of histone deacetylase activity by butyrate. J Nutr 2003;133:2485S-2493S

17. Gurvich N, Tsygankova OM, Meinkoth JL, Klein PS. Histone deacetylase is a target of valproic acid-mediated cellular differentiation. Cancer Res 2004;64:1079-1086.

18. Dalton WT, Jr., Ahearn MJ, McCredie KB, Freireich EJ, Stass SA, Trujillo JM. HL-60 cell line was derived from a patient with FAB-M2 and not FAB-M3. Blood 1988;71:242-247.

19. Jing Y, Xia L, Waxman S. Targeted removal of PML-RAR $\alpha$ protein is required prior to inhibition of histone deacetylase for overcoming all-trans retinoic acid differentiation resistance in acute promyelocytic leukemia. Blood 2002;100:1008-1013.

20. Melnick A, Licht JD. Deconstructing a disease: RAR $\alpha$, its fusion partners, and their roles in the pathogenesis of acute promyelocytic leukemia. Blood 1999;93:3167-3215. 